\title{
Patellar Shape is Associated With Femoral Trochlear Morphology in Individuals with Mature Skeletal Development
}

\section{Lanyu Qiu}

Department of Radiology, the First Affiliated Hospital of Chongqing Medical University Jia Li

Department of Radiology, the First Affiliated Hospital of Chongqing Medical University

\section{Bo Sheng}

Department of Radiology, the First Affiliated Hospital of Chongqing Medical University

\section{Haitao Yang}

Department of Radiology, the First Affiliated Hospital of Chongqing Medical University

\section{Fajin Lv}

Department of Radiology, the First Affiliated Hospital of Chongqing Medical University

\section{Zhibo Xiao}

Department of Radiology, the First Affiliated Hospital of Chongqing Medical University

Furong Lv ( $\square$ Ifr963@sina.com )

Department of Radiology, the First Affiliated Hospital of Chongqing Medical University

\section{Research Article}

Keywords: Patellar shape, Trochlear morphology, Patellofemoral instability, Knee surgery, Skeletal development

Posted Date: April 6th, 2021

DOI: https://doi.org/10.21203/rs.3.rs-361507/v1

License: (c) (1) This work is licensed under a Creative Commons Attribution 4.0 International License. Read Full License

Version of Record: A version of this preprint was published at BMC Musculoskeletal Disorders on January 17th, 2022. See the published version at https://doi.org/10.1186/s12891-022-05000-w. 


\section{Abstract}

Background: As several studies have detected correlations between patellar and femoral trochlear development, this raises the question of whether patellar shape is associated with trochlear developmental outcomes.

Methods: Patellar shape and femoral trochlear morphology were retrospectively analyzed in 240 subjects, of whom 80 each were classified as having Wiberg type I, II, and III patellae (groups A, B, and C, respectively). The sulcus angle (SA), lateral trochlea inclination angle (LTA), medial trochlear inclination angle (MTA), lateral facet length (LFL), medial facet length (MFL), lateral trochlear height (LTH), medial trochlear height $(\mathrm{MTH})$, trochlea sulcus height $(\mathrm{TH})$, and lateral-medial trochlear facet distance (TD) were analyzed as a means of evaluating trochlear morphology. Trochlear depth, trochlear condyle asymmetry, and trochlear facet asymmetry were additionally calculated, and differences in trochlear morphology and correlations between trochlear morphology and patellar shape were evaluated.

Results: The femoral trochlear parameters of patients in group A differed significantly from those of patients in groups B and C. No significant differences between groups B and C were evident. Patellar shape was positively correlated with LTA, MTA, MFL, trochlear index trochlear condyle asymmetry, and trochlear facet asymmetry, and was negatively correlated with SA.

Conclusions: These data indicated that patellar shape and trochlear morphology are related to one another. Relative to patients with Wiberg type II and III patellae, those with Wiberg type I patellae exhibited an increased trochlear inclination angle and a greater trochlear facet and condyle asymmetry, as well as a decreased SA.

Trial registration: Retrospectively registered

\section{Background}

The patellofemoral joint is a key knee component that is comprised of the patella, the anterior articular surface of the distal femur, and several soft tissues. Both active and passive mechanisms maintain patellofemoral joint stability, and many studies have explored the mechanics whereby the patella can track the trochlea during knee movement. Active and passive stability primarily arise through the actions of peripheral ligaments and muscles and as a consequence of the anatomical conformation of the patella and femoral trochlea, respectively [1]. The medial patellofemoral ligament (MPFL) is responsible for providing $50-60 \%$ of the restraint to patellar lateral displacement in full extension to $20^{\circ}$ flexion of the knee, thus serving as the main factor restricting patellar translation. Beyond this first $20^{\circ}$ range, however, the MPFL plays a less important role in patellar centering, with bony constraint instead being more important [2].

Patellofemoral instability occurs with high frequency in both adolescents and adults owing to a range of potential underlying anatomical abnormalities. Primary patella instability is generally treated 
conservatively, but reoccurrence affects an estimated $17-66 \%$ of patients, and is particularly common in those with trochlear dysplasia who exhibit a dislocation rate of up to $69 \%$ [3], necessitating surgical intervention. Surgical treatments focus on correcting any underlying anatomical abnormalities and bolstering intrinsic patellofemoral joint stability through approaches such as MPFL reconstruction, tibial tubercle transfer, patellar osteotomy, femoral and tibial osteotomies, and trochleoplasty. MPFL reconstruction is the most common of these approaches, and has achieved success in clinical contexts [1]. However, MPFL reconstruction can result in postoperative complications including recurrent patellar instability [4,5], primarily because such reconstruction does not correct underlying trochlear dysplasia, and trochlear shape is associated with recurrent patellar dislocation [3]. Trochleoplasty is generally only performed in individuals exhibiting severe trochlear dysplasia, despite recurrent patellar instability being very rare among patients undergoing this procedure. By restoring the underlying bony anatomy, trochleoplasty can significantly decrease postoperative instability [6]. Even so, the relative benefits of trochleoplasty and the appropriate means of performing this procedure remain controversial. It is thus essential to precisely define the differences in femoral trochlear morphology among patients and to understand how these morphological differences are related to different patellar shapes in order to guide trochleoplasty treatment planning.

Patellar and trochlear development has been widely studied in the context of patellar instability. Two primary theories exist with respect to the development of trochlear morphology. Several studies have found that trochlear sulcus shape is primarily determined during early embryonal stages $[7,8]$. However, other studies have suggested that trochlear and patellar development continues even after birth. Yang et al. [9] found that trochlear morphology shifts from normal to dysplastic when insufficient patellar stress was applied to the trochlea in animal model systems, and abnormal positioning of the patella relative to the femoral trochlear sulcus resulted in a more flattened sulcus [10]. Similar findings have also been observed in humans. Richmond et al. [11], for example, found that the patella and trochlea sulcus exhibit concurrent deepening over time prior to 8 years of age. Given that several studies have detected correlations between patellar and femoral trochlear development, this raises the question of whether patellar shape is associated with trochlear developmental outcomes. Bone shape and growth are associated with bone functions [12], and patella tracking is vital to joint development. A change in patellar shape may result in inappropriate trochlear sulcus modeling owing to the interaction between the two.

Trochleoplasty outcomes are associated with trochlear shape. However, correct patellar tracking is essential to facilitate the stability of the patellofemoral joint, suggesting that patellar shape should also be taken into consideration when conducting trochleoplasty. The goal of this study was to analyze femoral trochlear morphological differences associated with different patellar shapes and to study correlations between patellar shape and trochlear morphology. However, no studies to date have been published regarding the association between trochlear and patellar morphology in individuals with mature skeletal development. The aim of this study was therefore to address these limitations by studying a large cohort of patients with different patellar shapes in order to better understand these morphological relationships. 


\section{Methods}

\section{Study Population}

The Committee for Human Research of our institution approved the present retrospective study of patients that had undergone magnetic resonance imaging (MRI) scans for either knee pain or other medical examinations between January 2014 and August 202. Patients were excluded from this study if they (1) were < 19 years old; (2) had a history of knee surgery; (3) suffered from patellar dislocation; or (4) exhibited evidence of severe osteoarthritis ( $\geq K L 2$ grade) or inflammatory arthritis. MRI scans and medical records for all subjects were evaluated. The Wiberg classification system was used to divide patients into three groups according to patellar shape, with 80 Wiberg type I patients being incorporated into group A in this study, while 80 age-, gender- and side-matched patients with Wiberg type II and III patellae were each incorporated into groups $B$ and $C$, respectively. The patient selection process is outlined in Fig. 1.

\section{MRI Procols}

MRI scans were performed on a 1.5-T MRI equipment (Magnetom Essenza; Siemens Healthcare) with an extremity matrix knee coil (Tim coil) for all subjects. A standard MRI protocol was used, which included sagittal and coronal T1 turbo spin-echo (repetition time [TR], $306 \mathrm{~ms}$; echo time [TE], $12 \mathrm{~ms}$; field of view [FOV], $16 \mathrm{~cm}$; matrix size, $320 \times 320$; slice thickness, $4 \mathrm{~mm}$ ), sagittal T2 turbo spin-echo (TR, $3220 \mathrm{~ms}$; TE, $99 \mathrm{~ms}$; FOV, $16 \mathrm{~cm}$; matrix size, $320 \times 320$; slice thickness, $4 \mathrm{~mm}$ ), sagittal and coronal intermediateweighted turbo spin-echo with fat saturation (TR, $2800 \mathrm{~ms}$; TE, $38 \mathrm{~ms}$; FOV, $16 \mathrm{~cm}$; matrix size, $256 \times 256$; slice thickness, $4 \mathrm{~mm}$ ), and axial intermediate-weighted turbo spin-echo with fat saturation (TR, $2800 \mathrm{~ms}$; TE, $51 \mathrm{~ms}$; FOV, $16 \mathrm{~cm}$; matrix size, $232 \times 256$; slice thickness, $4 \mathrm{~mm}$ ).

\section{Image Analysis}

Two radiologists with 12 and 18 years of respective clinical experience who were blinded to this study conducted all image analyses. The Wiberg system $[13,14]$ was used to classify patellar shape (Fig. 2). Femoral trochlea parameters were measured on the transverse image obtained $3 \mathrm{~cm}$ above the femorotibial joint space where development of the femoral trochlea could be reliably evaluated [15]. Measured parameters included: the sulcus angle (SA), lateral trochlea inclination angle (LTA), medial trochlear inclination angle (MTA), lateral facet length (LFL), medial facet length (MFL), lateral trochlear height (LTH), medial trochlear height (MTH), trochlea sulcus height (TH), and lateral-medial trochlear facet distance (TD). Subsequent measurements were defined using the posterior condyle line (PCL) as a baseline. All measurements were based on bone, given that cartilaginous trochlear morphology differs from that of the underlying bony trochlea in those affected by trochlear dysplasia. The SA was assessed by measuring the angle between the line of lateral and medial facet inclination. The LTA and MTA were measured as angles between the facet inclination and the PCL. The LFL and MFL were measured based upon the distance between the most prominent point of the lateral and medial femoral condyle and the deepest point of the trochlear sulcus. The LTH and MTH were defined as the maximum anteroposterior distance of the lateral and medial femoral condyle and the PCL. The TH was defined as the minimum 
anteroposterior distance from the deepest point of the trochlear sulcus to the PCL. Trochlear depth was calculated as follows: depth $=(\mathrm{MTH}+\mathrm{LTH}) / 2-\mathrm{TH}$. Trochlear condyle asymmetry and trochlear facet asymmetry were respectively calculated according to the following formula [16]:TCA $=$ MTH/LTH $\times 100 \%$ and TFA $=$ MFH $/ \mathrm{LFH} \times 100 \%$.

\section{Statistical Analysis}

SPSSwas used for statistical testing. Categorical variables including sex and side were assessed using chi-squared tests. Continuous variables such as age and trochlear parameters were described using appropriate descriptive statistics, and, when normally distributed, were expressed as means \pm standard deviation and compared via one-way ANOVAs and least-significant difference tests. Relationships between patellar shape and trochlear morphology were assessed using Spearman's rank correlations. $\mathrm{P}<$ 0.05 was the significance threshold for this study. Intra- and interobserver reliability were assessed based on an intraclass correlation coefficient (ICCs) and 95\% confidence interval, with values of $0.8-0.9$ and > 0.9 being considered good and excellent, respectively.

\section{Results}

In total, 240 patients met with study inclusion and exclusion criteria. The ages of the three groups were $36.3 \pm 9.4$ years in group $A, 36.3 \pm 9.4$ years in group $B$, and $36.3 \pm 9.3$ years in group $C$. There were 5 left knee joints and 75 right knee joints in group $A, 6$ left knee joints and 74 right knee joints in group $B$, and 12 left knee joints and 68 right knee joints in group $C$. There were 37 women and 43 men in group A, 41 women and 39 men in group $B$, and 33 women and 47 men in group $C$. There were no significant differences in age, sex, or site between groups (Table 1). The intra-observer ICCs for the quantitative measurements ranged from $0.865-0.976$, whereas the inter-observer ICCs ranged from 0.869-0.983, consistent with good intra- and inter-reader reliability (Table 2). Significant differences in trochlear parameters were observed between group $A$ and groups $B$ and $C$ (Table 3 ). The SA of patients in group $A$ was $(138.9 \pm 9.5)^{\circ}$ and was smaller than that of patients in group $B(146.2 \pm 8.0)^{\circ}$ and group $C(146.5 \pm$ $8.5)^{\circ}(P<0.001)$. The inclination angles of the lateral and medial sides in group A were $(20.3 \pm 4.7)^{\circ}$ and $(20.8 \pm 7.3)^{\circ}$, respectively, with these values being greater than those in groups $B$ and $C$. The MFL in group A was $(16.9 \pm 2.5) \mathrm{mm}$ wider than that in groups $B$ and $C(P<0.001)$. Additionally, greater trochlear facet asymmetry, trochlear condyle asymmetry, and trochlear depth were observed in group $B(P<0.001)$ (Table 4). No trochlear parameters differed significantly between groups B and C. 
Table 1

Demographic factors of the patients.

\begin{tabular}{|llllll|}
\hline Characteristic & Group A & Group B & Group C & t value/ X2 value & P value \\
\hline Age, yr & $36.3 \pm 9.4$ & $36.3 \pm 9.4$ & $36.3 \pm 9.3$ & .006 & .994 \\
\hline Gender & & & & & \\
Male & 43 & 39 & 47 & 1.609 & .447 \\
\hline Female & 37 & 41 & 33 & & \\
Side & & & & & .126 \\
\hline Left & 5 & 6 & 12 & 4.135 & \\
\hline Right & 75 & 74 & 68 & & \\
\hline
\end{tabular}

Table 2

Inter- and intra-observer ICCs of parameters of trochlea morphology.

\begin{tabular}{|lll|}
\hline Measurement & Inter-observer ICCs & Intra-observer ICCs \\
\hline SA & $0.942(0.878-0.972)$ & $0.904(0.799-0.955)$ \\
\hline LTA & $0.902(0.795-0.953)$ & $0.886(0.761-0.946)$ \\
\hline MTA & $0.869(0.724-0.937)$ & $0.865(0.716-0.936)$ \\
\hline LFL & $0.930(0.854-0.967)$ & $0.951(0.898-0.977)$ \\
\hline MFL & $0.958(0.912-0.980)$ & $0.942(0.877-0.972)$ \\
\hline LTH & $0.983(0.965-0.992)$ & $0.975(0.947-0.988)$ \\
\hline TH & $0.983(0.965-0.992)$ & $0.974(0.949-0.988)$ \\
\hline MTH & $0.977(0.951-0.989)$ & $0.976(0.949-0.988)$ \\
\hline TD & $0.961(0.918-0.982)$ & $0.975(0.948-0.988)$ \\
\hline
\end{tabular}


Table 3

Results of morphometric parameters in the groups.

\begin{tabular}{|llllll|}
\hline Measurement & Group A & Group B & Group C & \multicolumn{2}{c|}{ Statistical analysis } \\
\hline SA $\left(^{\circ}\right)$ & & & & F & P \\
\hline LTA $\left(^{\circ}\right)$ & $20.3 \pm 4.7$ & $17.4 \pm 4.4$ & $17.2 \pm 4.7$ & 11.107 & .000 \\
\hline MTA $\left({ }^{\circ}\right)$ & $20.8 \pm 7.3$ & $16.3 \pm 6.7$ & $16.3 \pm 7.6$ & 10.167 & .000 \\
\hline LFL $(\mathrm{mm})$ & $22.2 \pm 2.2$ & $22.4 \pm 2.4$ & $22.6 \pm 2.6$ & .569 & .567 \\
\hline MFL $(\mathrm{mm})$ & $16.9 \pm 2.5$ & $15.0 \pm 2.6$ & $14.3 \pm 2.4$ & 21.761 & .000 \\
\hline LTH $(\mathrm{mm})$ & $57.3 \pm 4.6$ & $57.8 \pm 5.4$ & $57.6 \pm 5.0$ & .177 & .838 \\
\hline MTH $(\mathrm{mm})$ & $55.5 \pm 4.9$ & $54.8 \pm 4.9$ & $54.7 \pm 4.4$ & .730 & .483 \\
\hline TH $(\mathrm{mm})$ & $49.5 \pm 4.4$ & $50.8 \pm 4.7$ & $50.9 \pm 4.4$ & 2.201 & .113 \\
\hline TD $(\mathrm{mm})$ & $36.2 \pm 3.5$ & $35.3 \pm 3.3$ & $35.1 \pm 3.2$ & 2.303 & .102 \\
\hline
\end{tabular}

Table 4

Results of trochlear parameters in the groups.

\begin{tabular}{|lllllc|}
\hline Measurement & Group A & Group B & Group C & \multicolumn{2}{c|}{ Statistical analysis } \\
\hline & & & & $\mathrm{F}$ & $\mathrm{P}$ \\
\hline trochlear depth $(\mathrm{mm})$ & $6.9 \pm 1.8$ & $5.5 \pm 1.4$ & $5.2 \pm 1.7$ & 22.857 & .000 \\
\hline trochlear condyle asymmetry (\%) & $96.9 \pm 4.0$ & $95.0 \pm 4.1$ & $95.1 \pm 4.4$ & 5.341 & .005 \\
\hline trochlear facet asymmetry (\%) & $76.4 \pm 12.2$ & $67.5 \pm 12.3$ & $64.3 \pm 13.2$ & 19.600 & .000 \\
\hline
\end{tabular}

Patellar shape was significantly correlated with trochlear parameters including SA, LTA, MTA, MFL, trochlear depth, trochlear facet asymmetry, and trochlear condyle asymmetry.The symmetry of the patella medial and lateral articular surfaces differed between groups A, B, and C. Group A has the best symmetry, group $B$ was the second, and group $C$ exhibited the worst symmetry. Patellar facet symmetry was correlated with the aforementioned parameters and was not consistently associated with others. SA was inversely correlated with the patellar facet symmetry $(r=-0.348, P<0.001)$. Additionally, patellar facet symmetry was positively correlated with LTA $(r=0.274, P<0.001)$, MTA $(r=0.250, P<0.001)$, MFL $(r=$ $0.369, P<0.001)$, trochlear depth $(r=0.371, P<0.001)$, trochlear facet asymmetry $(r=0.378, P<0.001)$ and trochlear condyle asymmetry $(r=0.170, P=0.008)$.

\section{Discussion}


The primary finding of the present study was that there was a significant correlation between patellar shape and trochlear morphological parameters. The LTA, MTA, MFL, trochlear depth trochlear condyle asymmetry, and trochlear facet asymmetry were increased in subjects with Wiberg type I patellae relative to those with Wiberg type II and III patellae, whereas the opposite finding was observed for the SA in these patients. No significant differences in trochlear morphology were observed when comparing those with type II and III patellae. Correlation analyses revealed that individuals with a more symmetric patellar facet exhibited increased trochlear facet asymmetry and trochlear condyle asymmetry. This suggests an interaction between the patella and trochlear morphology during skeletal development, providing valuable information that can aid in selecting appropriate surgical procedures for treating patellofemoral joint instability.

The Wiberg classification system defines three primary patellar shapes based upon the size relationship between the medial and lateral patellar facets [13]. We observed different trochlear morphological parameters in those with Wiberg type I patellae relative to those with Wiberg type II and III patellae. Those with type I patellae exhibited equal medial and lateral facet lengths and the most symmetric facets. The MFL in group A was increased whereas the LFL was similar relative to other groups such that trochlear facet asymmetry (defined as MFL/LFL) was significantly increased. This suggests that trochlear and patellar morphology changed in a consistent manner across patients. In a study conducted by Fucentese et al. [14], patients with a dysplastic trochlea exhibited a smaller patellar size and an asymmetrical patellar facet. The SA was also evaluated in the present study, revealing that femoral trochlear dysplasia was reproducible and decreased in group A patients, explaining why type I patellae are more stable and associated with a lower incidence of patellar dislocation relative to type II and III patellae.

We detected correlations between patellar shape and femoral trochlear morphology, suggesting that the morphological parameters of these structures interact during development. Several studies have explored patellar and trochlear development, yielding inconsistnet findings. Some reports have suggested that congenital factors regulate femoral trochlear shape [7], whereas other studies have shown the trochlea and patella to develop continuously over time $[10,14,17,18]$. Fucentese et al. reported morphologic changes in the patellae of trochlear dysplasia patients, detecting molding activity between patellar and trochlear development [14]. In a study of skeletally immature cadavers, concurrent changes in patellar and trochlear shape were detected, consistent with our results and suggesting that these two structures may influence one another during development. We found patellar facet symmetry to be related to trochlear inclination and trochlear facet and condyle asymmetry, indicating that the medial patella is the primary portion that interacts with the trochlear. The mature patellae exhibited lateral facet predominance [19], whereas the medial and lateral patellar facets are equal in size during embryonic development, with medial trochlear morphology variability increasing with age [11]. Biedert et al. also found trochlear dysplasia was mainly located in the center and/or medial trochlea rather than the lateral trochlea [20].

In this study, we found that type I patellae, which exhibited nearly equal medial and lateral facet lengths, had more symmetrical trochlear parameters than type II or III patellae. Patients with increasing medial patellae facets exhibit a concurrent increase in trochlear morphological parameters, suggesting a 
relationship between patellofemoral structural and functional factors during development [17, 21]. Correct patellar tracking alters patellofemoral joint function and plays a key role in regulating knee joint stability. A deep sulcus and a high lateral trochlea can help to maintain normal patella tracking [6], while abnormal tracking can mold patellar and trochlear morphology and impact patellofemoral joint instability [22]. Causes of trochlear dysplasia may include developmental factors or false patellar tracking during childhood $[10,22]$. Stabilization produces changes throughout the knee range of movement, including both bony and soft tissue constraints. Bony constraint is particularly important after the first $20^{\circ}$ of knee flexion [2], while the patella begins to engage with the trochlea as the knee flexes to $30^{\circ}$. Poor patellar and trochlear tracking can give rise to trochlear and/or patellar dysplasia [11, 22]. In this report, we observed concurrent patellar and trochlear changes, possibly due to such patellar tracking during skeletal development.

Trochlear and patellar morphology serve to constrain patellofemoral joint instability, and can contribute to patellar dislocation. While trochleoplasty is an effective means of remodeling the trochlea to address patellar instability, it is rarely performed in clinical settings as it is a complex procedure and little information regarding its efficacy as a function of patellar and trochlear morphology is currently available. With sufficient morphological information, trochleoplasty may become a more viable means of addressing joint instability. In the present study, we found that the patellar and trochlear facets were matched in individuals with mature skeletal development such that a more symmetrical patella facet corresponded to a more symmetrical trochlear facet. It is important to take this into account when conducting trochleoplasty, as performing this procedure based on the shape of the patella and the trochlea rather than solely on the shape of the trochlea may reduce the risk of postoperative complications.

There are multiple limitations to this study. For one, we did not evaluate the position of the patella relative to the trochlea, and such positioning may impact the shape of these structures. Second, we did not measure patellar morphological parameters as this study was primarily focused on the relationship between patellar shape and trochlear morphology. Third, all measurements made herein were based on bony structures, and cartilaginous structures of the patellofemoral joint were not included. Finally, we only evaluated patients with mature skeletal development and thus cannot draw conclusions regarding whether the relationships between patellar shape and trochlear morphology arose during embryonic development or after birth. Future research will thus be needed to expand on this study and to address these points.

\section{Conclusions}

In summary, our data revealed a direct relationship between patellar shape and trochlear morphology. Developing a more comprehensive understanding of this relationship will enable clinicians to select suitable surgical procedures for the treatment of patients suffering from patellofemoral joint instability.

\section{List Of Abbreviations}


LFL lateral facet length,

LTA lateral trochlea inclination angle

LTH lateral trochlear height,

MFL medial facet length

MPFL medial patellofemoral ligament

MTA medial trochlear inclination angle

MTH medial trochlear height

PCL posterior condyle line

TD lateral-medial trochlear facet distance

TH trochlea sulcus height

SA sulcus angle

\section{Declarations}

\section{Ethics approval and consent to participate}

This study was approved by the Committee for Human of the First Affiliated Hospital of Chongqing Medical University. Individual consent for this retrospective analysis was waived which approved by the the Committee for Human of the First Affiliated Hospital of Chongqing Medical University. All methods were carried out in accordance with relevant guidelines and regulations.

\section{Consent for publication}

Not applicable.

\section{Availability of data and materials}

The datasets used and/or analysed during the current study are available from the corresponding author on reasonable request.

\section{Competing interests}

The authors declare that they have no competing interests.

\section{Funding}


This study was supported by Science and Technology Commission Project of Chongqing. (Grant No. cstc2018jscx-mszdX0042)

\section{Authors' contributions}

LQ and $\mathrm{JL}$ analyzed and interpreted the patient data regarding the MRI measurement parameters. LQ, JL and FLwas a major contributor in writing the manuscript. BS and ZX observed and recorded the status of patellar shape. HY and FL designed the work. All authors read and approved the final manuscript.

\section{Acknowledgements}

None.

\section{References}

1. Dejour D, Le Coultre B Osteotomies in Patello-Femoral Instabilities. Sports Med Arthrosc Rev. 2018,26(1):8-15.

2. Bollier M, Fulkerson JP The role of trochlear dysplasia in patellofemoral instability. J Am Acad Orthop Surg. 2011,19(1):8-16.

3. Lewallen LW, Mclntosh AL, Dahm DL Predictors of recurrent instability after acute patellofemoral dislocation in pediatric and adolescent patients. Am J Sports Med. 2013,41(3):575-81.

4. Van Huyssteen AL, Hendrix MR, Barnett AJ, et al Cartilage-bone mismatch in the dysplastic trochlea. An MRI study. J Bone Joint Surg Br. 2006,88(5):688-91.

5. Rajdev NR, Parikh SN Femoral trochlea does not remodel after patellar stabilization in children older than 10 years of age. J Pediatr Orthop B. 2019,28(2):139-143.

6. Carstensen SE, Menzer HM, Diduch DR Patellar Instability: When is Trochleoplasty Necessary? Sports Med Arthrosc Rev. 2017,25(2):92-99.

7. Øye CR, Holen KJ, Foss OA Mapping of the femoral trochlea in a newborn population: an ultrasonographic study. Acta Radiol. 2015,56(2):234-43

8. Øye CR, Foss OA, Holen KJ Minor change in the sulcus angle during the first six years of life: a prospective study of the femoral trochlea development in dysplastic and normal knees. J Child Orthop. 2018,12(3):245-250.

9. Yang G, Li F, Lu J, et al The dysplastic trochlear sulcus due to the insufficient patellar stress in growing rats. BMC Musculoskelet Disord. 2019,20(1) 411

10. Huri G, Atay OA, Ergen B, et al Development of femoral trochlear groove in growing rabbit after patellar instability. Knee Surg Sports Traumatol Arthrosc. 2012,20(2):232-8.

11. Richmond CG, Shea KG, Burlile JF, et al Patellar-Trochlear Morphology in Pediatric Patients From 2 to 11 Years of Age: A Descriptive Analysis Based on Computed Tomography Scanning. J Pediatr Orthop. 2020; 40(2):96-102. 
12. Mittag U, Kriechbaumer A, Bartsch $M$, Rittweger $J$ Form follows function: a computational simulation exercise on bone shape forming and conservation. J Musculoskelet Neuronal Interact. 2015,15(2) :15-26

13. Wiberg G Roentgenographic and anatomic studies on the femoropatellar joint. Acta Orthop Scand. 1941,12:319 - 410.

14. Fucentese SF, von Roll A, Koch PP,et al The patella morphology in trochlear dysplasia-a comparative MRI study. Knee. 2006,13(2):145-50.

15. Pfirrmann CW, Zanetti M, Romero J, et al Femoral trochlear dysplasia: MR findings. Radiology. 2000,216(3):858-64.

16. Arendt EA, England K, Agel J,et al An analysis of knee anatomic imaging factors associated with primary lateral patellar dislocations. Knee Surg Sports Traumatol Arthrosc. 2017,25(10):3099-3107.

17. Ferlic PW, Runer A, Dammerer D,et al Patella Height Correlates With Trochlear Dysplasia: A Computed Tomography Image Analysis. Arthroscopy. 2018,34(6):1921-1928.

18. Peeters W, Van Haver A, Van den Wyngaert S,et al A landmark-based $3 D$ analysis reveals a narrower tibial plateau and patella in trochlear dysplastic knees. Knee Surg Sports Traumatol Arthrosc. 2020,28(7):2224-2232.

19. Fox AJ, Wanivenhaus F, Rodeo SA The basic science of the patella: structure, composition, and function. J Knee Surg. 2012,25(2):127-41.

20. Biedert RM, Bachmann M Anterior-posterior trochlear measurements of normal and dysplastic trochlea by axial magnetic resonance imaging. Knee Surg Sports Traumatol Arthrosc. 2009,17(10):1225-30.

21. Levy BJ, Tanaka MJ, Fulkerson JP Current Concepts Regarding Patellofemoral Trochlear Dysplasia. Am J Sports Med. 6:363546520958423.

22. Kaiser $P$, Loth $F$, Attal $R$, et al Static patella tilt and axial engagement in knee extension are mainly influenced by knee torsion, the tibial tubercle-trochlear groove distance (TTTG), and trochlear dysplasia but not by femoral or tibial torsion. Knee Surg Sports Traumatol Arthrosc. 2020,28(3):952959

\section{Figures}




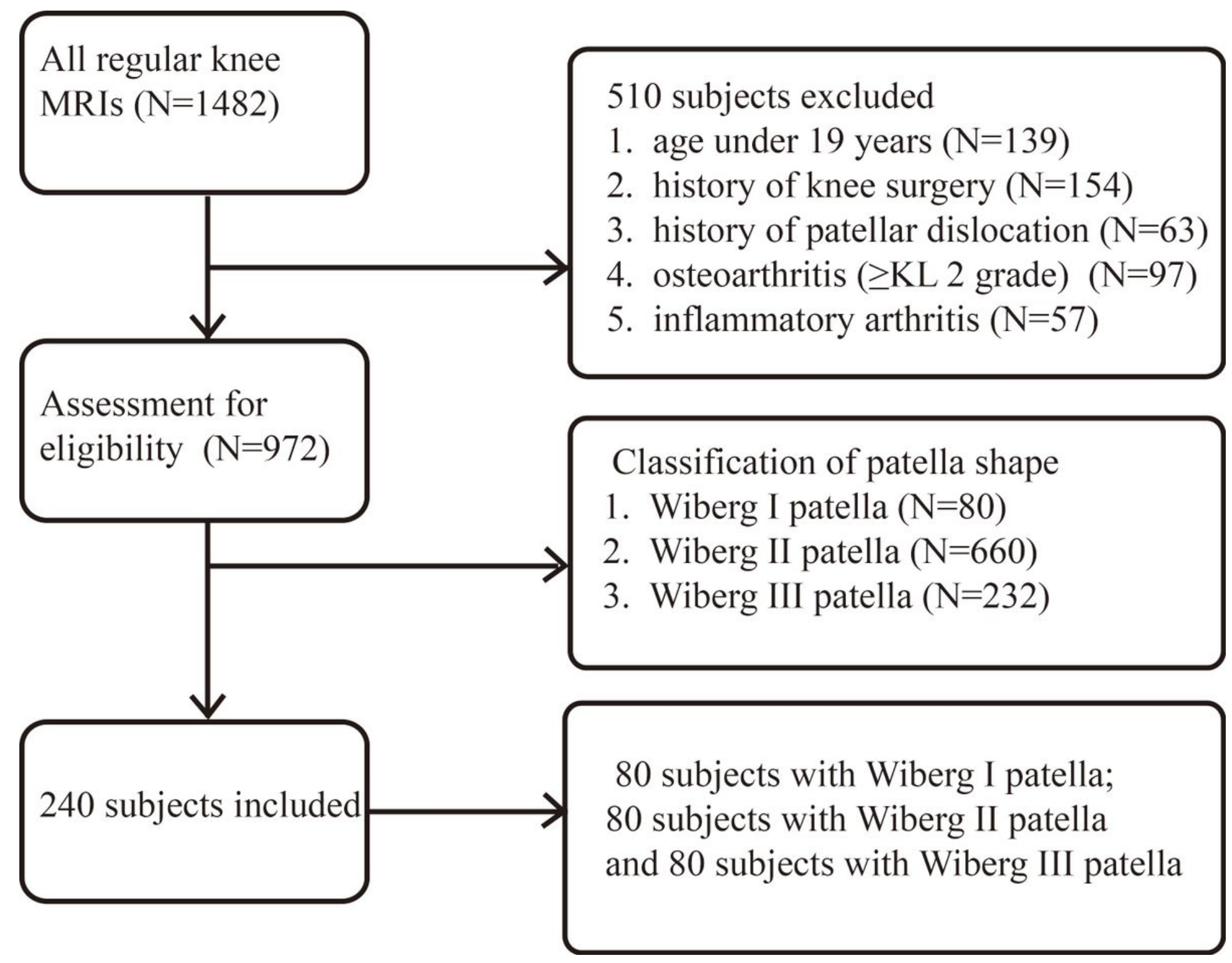

Figure 1

Flowchart for subject selection.
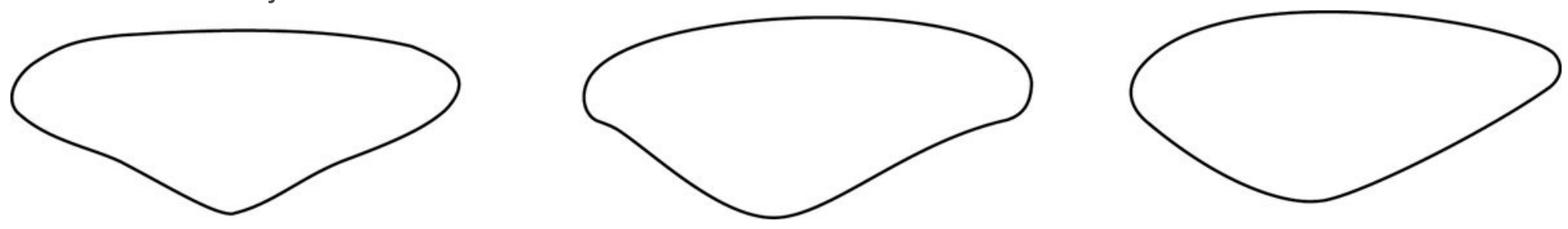

Figure 2

Wiberg classification for patella morphology $[13,14]$. Wiberg type I patella (the left):medial facet equal size as lateral facet; Wiberg type II patella (the center) : medial facet smaller than lateral facet; Wiberg type III patella (the right) : medial facet very much smaller than lateral facet. 

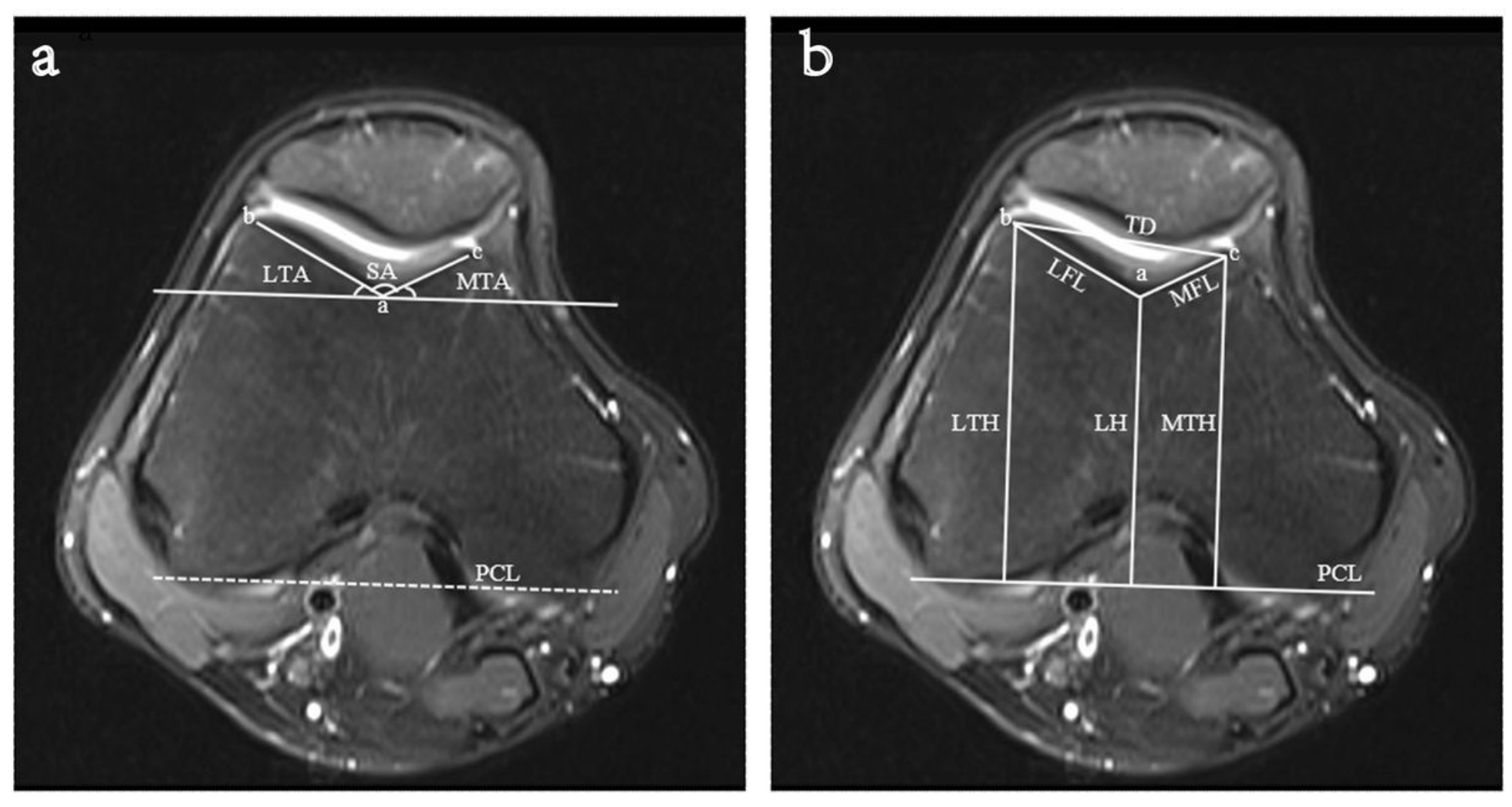

Figure 3

Measurements on the transverse image obtained $3 \mathrm{~cm}$ above the femorotibial joint space. (A) The angle of trochlear facet. The SA was the angle between the $a b$ and ac; LTA and MTA were the angle between the posterior condyle line (PCL) and the $a b$ and ac respectively. $a$ : the deepest point of the trochlear sulcus; $b$ and $\mathrm{c}$ : the point of the most prominent point of the lateral and medial femoral condyle. (B) The parameters of trochlear morphology. LFL was the distance between a and b. MFL was the distance between a and c. TD was the distance between $b$ and c. LH, LTH and MTH were the vertical distance from $a, b$ and $c$ to PCL. Note: LFL lateral facet length, LTA lateral trochlea inclination angle, LTH lateral trochlear height, MFL medial facet length, MPFL medial patellofemoral ligament, MTA medial trochlear inclination angle, MTH medial trochlear height PCL posterior condyle line, TD lateral-medial trochlear facet distance, TH trochlea sulcus height, SA sulcus angle 